\title{
The spectral-type/luminosity and the spectral-type/satellite-density relations in the 2dFGRS
}

\author{
B. Kelm ${ }^{1}$, P. Focardi ${ }^{1}$, and G. Sorrentino ${ }^{2}$ \\ 1 Dipartimento di Astronomia, Università di Bologna, V. Ranzani 1, 40127 Bologna, Italy \\ e-mail: birgit.kelm@unibo.it \\ 2 INAF - Osservatorio Astronomico di Capodimonte, V. Moiariello 16, 80131 Napoli, Italy \\ Received 10 June 2004 / Accepted 19 June 2005
}

ABSTRACT

We examine the relative fractions of passive (type 1), quiet-SF (type 2) and active-SF (type $3+4$ ) galaxies as a function of luminosity and number of neighbours in several volume-limited samples selected from the 2dFGRS. Neighbours are counted within $1 h_{75}^{-1}$ Mpc projected distance and $\pm 1000 \mathrm{~km} \mathrm{~s}^{-1}$ depth. We apply a maximum magnitude difference criterion and require neighbours to be fainter than the galaxy itself. We show that, whatever the environment, passive galaxies dominate in bright samples and active-SF galaxies in faint samples, whereas quiet-SF galaxies never dominate. We further show that in bright samples $\left(M_{B}-5 \log h_{75} \leq-19\right)$ the fraction of passive galaxies grows steadily with fainter neighbour density, whereas in faint samples a threshold-like dependence is observed. This suggests that the spectral-type/density $(\approx$ morphology/density) relation extends to the intermediate dense environment, but only in the surroundings of luminous galaxies and that it reflects an enhancement of the number of satellites rather than stronger clustering among galaxies themselves. Our analysis indicates that, in general, luminosity is a good tracer of galaxy halo mass and that it dominates over environment (satellite density) in setting the spectral type mix of a population. However, minority populations exist, such as luminous SF galaxies and faint passive galaxies, whose luminosity is an inaccurate tracer of halo mass.

Key words. galaxies: general - galaxies: fundamental parameters - galaxies: stellar content - galaxies: statistics galaxies: clusters: general

\section{Introduction}

The existence of a relation between galaxy type and environment dates back to pioneering work by Hubble \& Humason (1931) and was first quantified by Davis \& Geller (1976) and Dressler (1980). Dressler found that, in nearby clusters, the fraction of elliptical galaxies increases and the fraction of spirals decreases with increasing density. Whitmore \& Gilmore (1991) and Whitmore et al. (1993) suggested that the correlation between morphology and cluster-centric-radius is tighter than the correlation between morphology and density. Deep surveys such as the 2dFGRS and the SDSS have confirmed that the star-formation level in galaxies decreases at large galaxy density (Lewis et al. 2002; Gomez et al. 2003; Goto et al. 2002，2003; Balogh et al. 2004a; Christlein \& Zabludoff 2005) and that a threshold is reached at low densities ( $\approx 1$ gal Mpc $\left.{ }^{-2}, M_{B} \leq-19\right)$, below which no further increase in star-formation is observed. These results provide evidence that, at least at low redshift, a strong correlation exists between the characteristics of the stellar component of a galaxy and its surrounding environment, with luminosity and color being the galaxy properties most strongly correlated with environment (Kauffmann et al. 2004; Blanton et al. 2005a). But the exact dependence of the morphology/density relation on density and on luminosity is still a matter of debate. It is not clear yet whether the relation extends to galaxies in systems less dense than clusters (i.e. to the majority of galaxies). Postman \& Geller (1984) and Maia \& da Costa (1990) claim that the morphology/density relation certainly extends to groups, whereas Whitmore (1995) does not confirm their result. Tran et al. (2001) and Helsdon \& Ponman (2003) support the existence of the morphology/density relation in X-ray bright groups. Dominguez et al. (2002) show that the relation is observed in very massive (optically selected) groups only, whereas Kelm \& Focardi (2004a) report that the frequency of early-type galaxies is larger in Compact Groups than among isolated galaxies. Mateus \& Sodré (2004) and Gerken et al. (2004) provide evidence that, even outside clusters, star formation properties are affected in all ranges of density. Tanaka et al. (2004) find that only faint galaxies show a break in star formation and morphology at a critical local density. Also the role of luminosity on the morphology/density relation has still to be fully disentangled. Galaxy clustering appears to depend on luminosity for luminous galaxies and on color for low luminous ones (Norberg et al. 2002a; Hogg et al. 2003; Balogh et al. 2004b; Berlind et al. 2005; Zehavi et al. 2005). For blue galaxies the relation between environment; and luminosity is typically weak, whereas for red galaxies clustering is likely a nonmonotonic function of luminosity, peaking at both high and low luminosities. 
In this paper we investigate the relation linking the galaxy spectral type mix with both luminosity and local environment. Specifically we explore 1) how the spectral-type/luminosity relation varies as a function of environment; and 2) how the spectral-type/satellite-density relation varies as a function of luminosity. We use data from the $2 \mathrm{dF}$ to select 10 different volume limited samples, covering a wide luminosity range $\left(-22.5 \leq M_{B}-5 \log h_{75} \leq-17.0\right)$. We evaluate for each galaxy the neighbour density on the characteristic scale of galaxy groups $\left(\sim 1 h_{75}^{-1} \mathrm{Mpc}\right)$ which further corresponds to the present day typical virial radius of halos. Galaxy properties are expected to correlate most strongly with densities evaluated on this scale, also from a theoretical standpoint (Blanton et al. 2005b; Kauffmann et al. 2004; Berlind et al. 2005).

At variance with previous analysis we compute neighbour density applying a maximum magnitude difference criterion and count neighbours over a 2 mag interval. The adopted range in magnitude reduces the number of galaxies that have no neighbours, on a group scale, to $\simeq 15 \%$ and associates most galaxies $(2 / 3)$ with a number of neighbours $(1 \leq$ neigh $\leq 8)$ that matches the typical observed environment of galaxies in groups. We also limit neighbour computation to galaxies that are fainter than (or equally luminous to) the galaxy itself. Usually, when computing density in volume-limited samples, no distinction is made between brighter and fainter neighbours. This implies that, within the same volume-limited sample, the density definition depends on luminosity: the environment of luminous galaxies is defined by fainter neighbours whereas the environment of low luminous galaxies is defined by brighter neighbours. But, obviously, the impact of a brighter or a fainter companion on a galaxy is different. Less massive companions have likely been, or will be, accreted by the galaxy halo, whereas more massive companions will likely accrete the galaxy and destroy its halo.

Our density definition is luminosity-independent. For luminous galaxies, the environment on the $1 h_{75}^{-1} \mathrm{Mpc}$ scale is likely to correspond to the density of satellites that have been captured by the galaxy halo. Conversely, for low luminous galaxies, it likely corresponds to the clustering of small halos among themselves, or, in the case of a galaxy swallowed up in the halo of a bigger companion, for the richness of satellites within this large halo.

In Sect. 2 we present the sample, in Sect. 3 we discuss the link between density distribution and luminosity. In Sects. 4 and 5 we explore the dependence on density of the spectraltype/luminosity relation. In Sect. 6 we investigate the dependence on luminosity of the spectral-type/density relation. The summary and conclusions are given in Sect. 7 . We assume $\Omega_{\mathrm{M}}=0.3, \Omega_{\Lambda}=0.7$, and $h_{75}=H_{0} /\left(75 \mathrm{~km} \mathrm{~s}^{-1} \mathrm{Mpc}^{-1}\right)=1$.

\section{The sample}

The sample we use for the present analysis is selected from the 2dFGRS (Colless et al. 2001, Colless et al. 2003). The $2 \mathrm{dF}$ covers $\sim 1800$ square degrees and is complete for galaxies down to an extinction-corrected limit of $b_{J}=19.45$. It provides redshifts, in the range $0 \leq z \leq 0.3$, for 221, 496 galaxies selected from the APM catalogue (Norberg et al. 2002b), which is 90-95\% complete (Maddox et al. 1990). Because saturation effects and stellar contamination cannot be ignored for bright galaxies, we exclude from the sample galaxies brighter than $b_{j}=16$.

Each $2 \mathrm{dF}$ galaxy spectrum is typed on the basis of the relative strength of its first two principal components (for details on the PCA see Folkes et al. 1999), which are the emission and absorption components within the spectrum. The parameter $\eta$ (Madgwick et al. 2002) is the linear combination of these two components. Qualitatively $\eta$ is an indicator of the ratio of the present to the past star-formation activity of each galaxy, but it is reliable only for $z \leq 0.15$. Clusters are dominated by galaxies with the lowest $\eta$ values, whereas the field contains a much larger proportion of galaxies with higher $(\eta>0)$ values. The median $\eta$ correlates with morphological classes, (low $\eta$ are typically early type galaxies, high $\eta$ late type galaxies) although there is a large scatter in the $\eta$ values of spectra that lie within a given morphological class.

As in Madgwick et al. (2002) we divide the $\eta$ scale into 4 intervals:

$$
\begin{aligned}
& \eta<-1.4 \Longrightarrow \text { Type } 1 \\
& -1.4 \leq \eta<1.1 \Longrightarrow \text { Type } 2 \\
& 1.1 \leq \eta<3.5 \Longrightarrow \text { Type } 3
\end{aligned}
$$

$\eta \geq 3.5 \Longrightarrow$ Type 4 .

We group together type 3 and type 4 galaxies, the latter being rare. Throughout the paper, type 1, type 2 and type 3 + type 4 galaxies are named passive, quiet-SF and active$\mathrm{SF}$ respectively. We keep quiet-SF and active-SF galaxies separate, in order to investigate any dependence of SF triggering processes on specific density characteristics.

For each $2 \mathrm{dF}$ galaxy (random fields excluded) with $z \leq 0.15$ we have automatically identified neighbours within $1 h_{75}^{-1} \mathrm{Mpc}$ projected distance and $\pm 1000 \mathrm{~km} \mathrm{~s}^{-1}$ depth. We count as neighbours all galaxies fainter than the galaxy itself that satisfy a maximum magnitude difference criterion $\left(-2 \leq M_{\mathrm{gal}}-\right.$ $\left.M_{\text {neigh }} \leq 0\right)$. We reject from the sample all galaxies whose 2 magnitude fainter companions would fail the $2 \mathrm{dF}$ selection criteria. The minimum fiber separation of the $2 \mathrm{dF}$ survey $\left(\sim 30^{\prime \prime}\right)$ tends to reduce the number of close neighbours of galaxies, a bias that might affect passive galaxies more severely than SF galaxies. However, the bias is likely marginal as this separation corresponds to less than one-tenth of the explored distance even for the highest redshift galaxies.

The final sample includes $\sim 14000$ galaxies in the redshift range $0.0156 \leq z \leq 0.15$, and absolute magnitude range $-22.5 \leq M_{B}-5 \log h_{75} \leq-17.0$. Absolute magnitudes are computed adopting the k-correction as in Magdwick et al. (2002), which varies with galaxy spectral-type. We split the sample into 10 different volume-limited subsamples, covering a 1 mag range each and overlapping by $0.5 \mathrm{mag}$. Since the $\mathrm{k}$-corrections are class dependent, the $z_{\min }$ and $z_{\max }$ values corresponding to a given absolute magnitude range are also class dependent. Hence, the volumes defining the samples for two 
Table 1. Spectral-type content of the volume limited samples. Each sample spans a one magnitude range, and overlaps by 0.5 mag with the next one. $M_{B}$ corresponds to the central value of the magnitude in each bin.

\begin{tabular}{rrrrr}
\hline \hline$M_{\mathrm{B}}$ & $N_{\mathrm{T} 1}$ & $N_{\mathrm{T} 2}$ & $N_{\mathrm{T} 3+\mathrm{T} 4}$ & $N_{\text {tot }}$ \\
\hline-22.0 & 782 & 249 & 41 & 1072 \\
-21.5 & 1865 & 699 & 144 & 2708 \\
-21.0 & 2703 & 1395 & 421 & 4519 \\
-20.5 & 2732 & 1908 & 828 & 5468 \\
-20.0 & 2134 & 1860 & 1100 & 5094 \\
-19.5 & 1194 & 1183 & 918 & 3295 \\
-19.0 & 543 & 692 & 773 & 2008 \\
-18.5 & 277 & 467 & 663 & 1407 \\
-18.0 & 158 & 327 & 451 & 936 \\
-17.5 & 77 & 189 & 276 & 542 \\
\hline
\end{tabular}

different spectral classes, for the same bin in absolute magnitude, will not exactly coincide (see also Norberg et al. 2002a).

Table 1 lists the spectral-type composition in each volume limited sample. The gap between galaxies in the faintest and the brightest volume limited samples corresponds to a factor $\sim 150$ in luminosity.

\section{The dependence on luminosity of the satellite-density distribution}

We assign a local density (number of fainter neighbours within $1 h_{75}^{-1} \mathrm{Mpc}, \pm 1000 \mathrm{~km}^{-1}$ depth and a $2-\Delta \mathrm{Mag}$ range) to all galaxies in our sample. We then define four distinct density regimes characterized by different number of neighbours:

neigh $=0$

$1 \leq$ neigh $\leq 2$

$3 \leq$ neigh $\leq 8$

neigh $>8$.

Table 2 lists the number of galaxies per spectral-type in the four defined density ranges and for each volume limited sample. The density parameterization is such that most galaxies $(\sim 2 / 3)$ are in the two central bins, which exhibit the typical galaxy density of groups. Obviously, computing densities on the galaxy group scale does not correspond to selecting a sample of $2 \mathrm{dF}$ groups (Eke et al. 2004a,b; Merchán \& Zandivarez 2002).

The relation linking luminosity and fainter neighbour density is shown in Fig. 1. Distributions of passive, quiet-SF, active-SF and all-type galaxies, normalized to the total number of galaxies of a given type, are shown, for the 10 volumelimited samples. The last column of Fig. 1 shows that in a composite (all-type) population, the number of fainter neighbours associated with galaxies is a weak function of luminosity. This recalls the result by Zehavi et al. (2002), showing that all-type galaxy subsamples in 3 distinct absolute magnitude ranges have real-space correlation functions that are parallel power-laws.
If we assume that for luminous galaxies, the environment on a $1 h_{75}^{-1}$ Mpc scale essentially stands for the density of satellites that have been captured by the galaxy halo whereas for faint galaxies, it stands for the number of neighbour galaxies still in their own small halo, then Fig. 1 actually indicates that the distribution of satellites surrounding luminous central galaxies within large halos, and the distribution of fainter companions surrounding low luminous galaxies, are almost self similar. This implies that without information on the luminosity of the galaxies, the neighbour density distribution of galaxies (on $1 h_{75}^{-1} \mathrm{Mpc}$ scale) cannot be used to discriminate between massive group-size halos and associations of galaxies in distinct small-size halos.

Figure 1 also clearly shows that at all luminosities, passive and SF galaxies exhibit different distributions and that the excess of companions surrounding passive galaxies is not limited to luminous galaxies (Norberg et al. 2002a; Hogg et al. 2003; Berlind et al. 2005; Blanton et al. 2005a) but is instead a general characteristic of passive galaxies. The all-type galaxy distribution reflects the passive population at the bright end, and the star-forming galaxy population at the faint end. Our assumption that luminous galaxies are central galaxies within group-size halos and faint galaxies are central galaxies within small size halos is therefore further consistent with the expectation that the SFR of a galaxy is a decreasing function of its halo mass.

However SF galaxies are found among bright galaxies and passive galaxies among faint ones. How can we explain their existence? We will assume that luminous SF galaxies are hosted in small mass halos; they may exhibit several neighbours, but, at variance with luminous passive galaxies, neighbours are not embedded within the galaxy halo. As a consequence optically selected passive dominated groups are predicted to be systematically more massive than optically selected SF dominated groups (Kelm \& Focardi 2004a,b; Mulchaey et al. 2003). Similarly, we explain the existence of low luminous passive galaxies assuming that they are satellites embedded within the halo of a large (group-size) system. Actually, the large fraction of faint passive galaxies with $>8$ neighbours among faint galaxies (see Table 2) suggests that this population is tracing a large potential well (Norberg et al. 2002a; Hogg et al. 2003; Berlind et al. 2005; Zehavi et al. 2005; Jing \& Borner 2004), with the galaxy and all of its fainter neighbours having been accreted by a massive system.

The stronger clustering of passive galaxies relative to SF galaxies, on the group scale, appears to arise from two distinct contributions. At the luminous end, it is due to an excess of satellites surrounding central galaxies inside large halos. At the faint end it is due to an excess of satellites that are strongly correlated among themselves.

\section{The dependence on satellite-density of the spectral-type/luminosity relation}

Figure 2 shows the fractions of passive, quiet-SF and active-SF galaxies in the 10 volume-limited samples for the total (all neighbour density) population. It is a global 
Table 2. Spectral-type content of the volume-limited samples as a function of neighbour density.

\begin{tabular}{|c|c|c|c|c|c|c|c|c|c|c|c|c|c|c|c|c|}
\hline \multirow[b]{2}{*}{$M_{B}$} & \multicolumn{4}{|c|}{ neigh $=0$} & \multicolumn{4}{|c|}{$1 \leq$ neigh $\leq 2$} & \multicolumn{4}{|c|}{$3 \leq$ neigh $\leq 8$} & \multicolumn{4}{|c|}{ neigh $>8$} \\
\hline & $T_{1}$ & $T_{2}$ & $T_{3+4}$ & $\Sigma$ & $T_{1}$ & $T_{2}$ & $T_{3+4}$ & $\Sigma$ & $T_{1}$ & $T_{2}$ & $T_{3+4}$ & $\Sigma$ & $T_{1}$ & $T_{2}$ & $T_{3+4}$ & $\Sigma$ \\
\hline-22.0 & 153 & 94 & 17 & 264 & 297 & 102 & 14 & 413 & 281 & 52 & 10 & 343 & 51 & 1 & 0 & 52 \\
\hline-21.5 & 348 & 213 & 39 & 600 & 649 & 288 & 65 & 1002 & 684 & 175 & 40 & 899 & 184 & 23 & 0 & 207 \\
\hline-21.0 & 466 & 344 & 92 & 902 & 823 & 549 & 165 & 1537 & 1022 & 425 & 142 & 1589 & 392 & 77 & 22 & 491 \\
\hline-20.5 & 398 & 436 & 166 & 1000 & 830 & 701 & 325 & 1856 & 1057 & 641 & 279 & 1977 & 447 & 130 & 58 & 635 \\
\hline-20.0 & 254 & 427 & 206 & 887 & 652 & 656 & 445 & 1753 & 817 & 606 & 361 & 1784 & 411 & 171 & 88 & 670 \\
\hline-19.5 & 139 & 250 & 181 & 570 & 346 & 424 & 353 & 1123 & 441 & 377 & 303 & 1121 & 268 & 132 & 81 & 481 \\
\hline-19.0 & 81 & 147 & 153 & 381 & 157 & 255 & 280 & 692 & 177 & 217 & 264 & 658 & 128 & 73 & 76 & 277 \\
\hline-18.5 & 40 & 80 & 114 & 234 & 73 & 156 & 265 & 494 & 81 & 161 & 218 & 460 & 83 & 70 & 66 & 219 \\
\hline-18.0 & 15 & 42 & 71 & 128 & 33 & 107 & 159 & 299 & 44 & 114 & 163 & 321 & 66 & 64 & 58 & 188 \\
\hline-17.5 & 4 & 23 & 48 & 75 & 7 & 55 & 84 & 146 & 26 & 77 & 107 & 210 & 40 & 34 & 37 & 111 \\
\hline
\end{tabular}

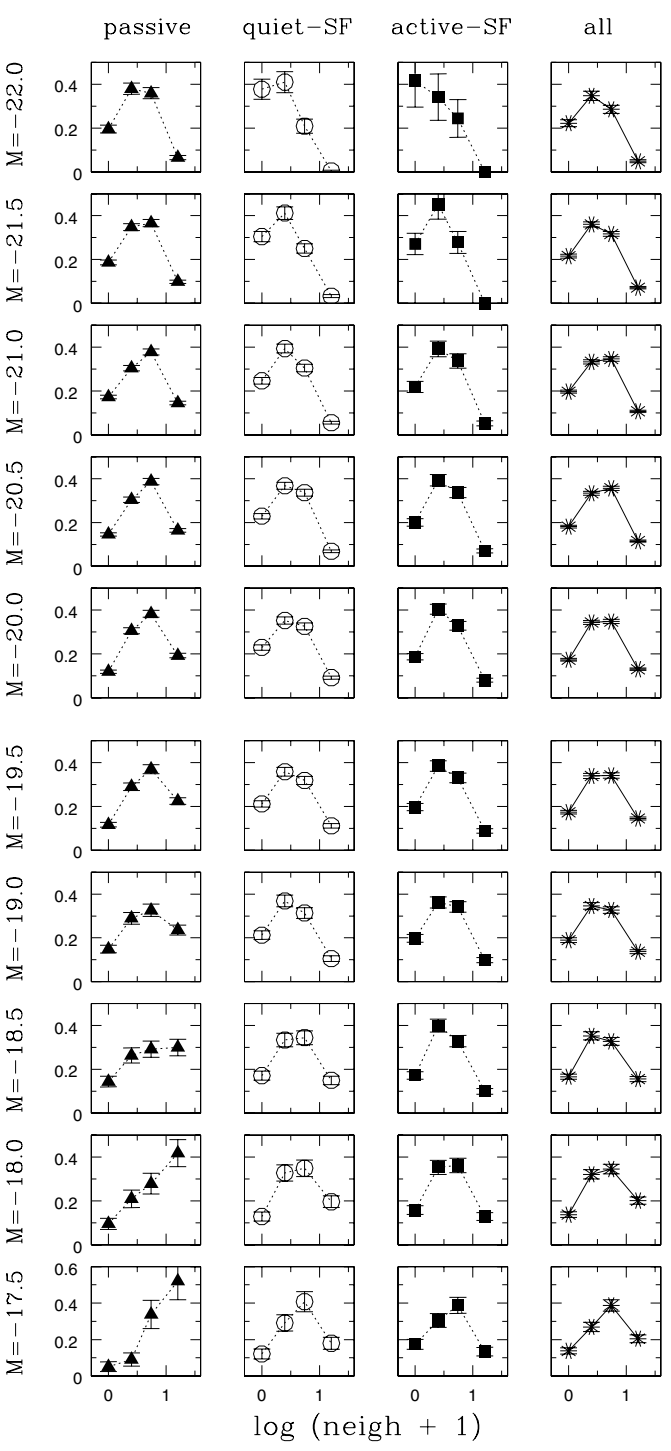

Fig. 1. Relative distributions of passive (type 1), quiet-SF (type 2), active-SF (type $3+4$ ) and all-type galaxies as a function of number of fainter neighbours (in 4 intervals), for the 10 volume limited samples. Numbers are normalized to the total number of galaxies of a given type: for each spectral type the sum over all environments is 1 . The number of neighbour distribution, in the all-type galaxy sample, undergoes no significant modification with luminosity.

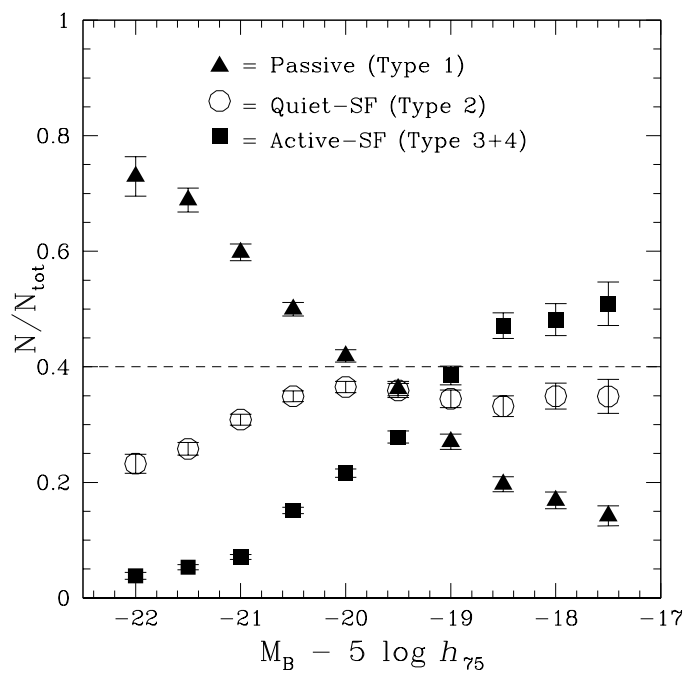

Fig. 2. The relative content in passive, quiet-SF and active-SF galaxies in the 10 volume-limited samples. Error bars are multinomial. The $40 \%$ fraction line is drawn to reveal "dominant" populations. Passive galaxies dominate in bright samples, active-SF galaxies in faint samples, quiet-SF galaxies are never dominant. The contribution of the 3 populations is comparable in the $M_{B}-5 \log h_{75} \in$ $[-20 \div-19]$ range.

spectral-type/luminosity plot that indicates how the fraction of passive and SF galaxies varies as a function of luminosity. It is similar to Fig. 9 in Norberg et al. (2002a), in which fractions have been derived for late-type and early-type galaxies only.

The horizontal line in Fig. 2 denotes the $40 \%$ fraction: points above this threshold mark dominant populations. Passive galaxies are "dominant" in galaxy samples brighter than $M_{B}-$ $5 \log h_{75}=-20$, active-SF galaxies are "dominant" in samples fainter than $M_{B}-5 \log h_{75}=-19$. Contributions from different spectral-type populations are comparable in the $[-20 \div-19]$ magnitude bin. These trends confirm that star-formation activity in the local universe definitely is a characteristic of low luminosity galaxies.

In Fig. 3 we break down the contributions of passive and SF galaxies to the Fig. 2 plot into their contributions from systems exhibiting different numbers of fainter neighbours. This allows us to explore how strongly the relative fraction of passive and 


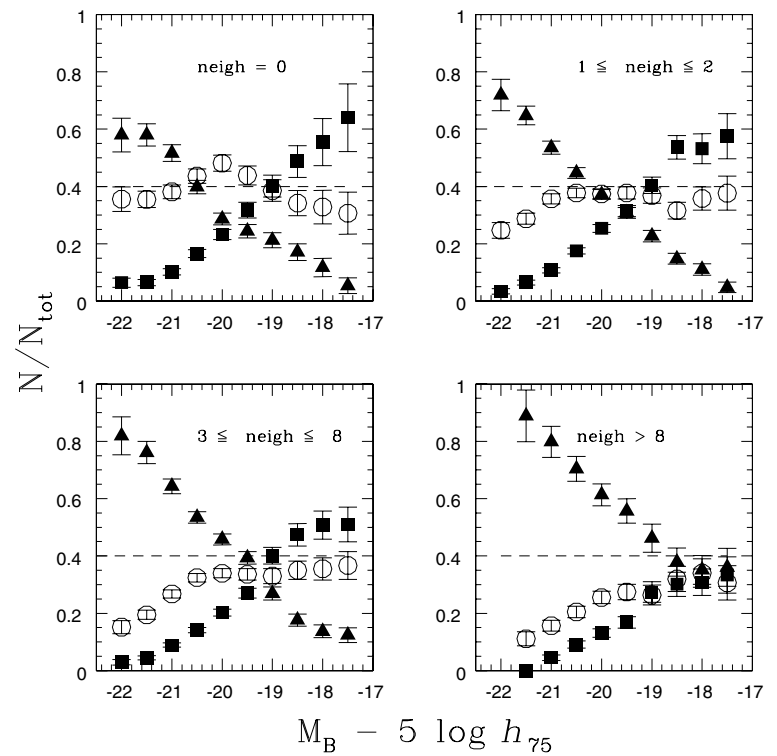

Fig. 3. Relative content in passive, quiet-SF and active-SF galaxies in the 10 volume-limited samples (symbols as in Fig. 2). Each panel refers to galaxies in a specific neighbour density bin. The absence of satellites $($ neigh $=0)$ efficiently reduces the dominance of passive galaxies in luminous samples, raising the contribution of quiet-SF galaxies. Conversely, a high density environment (neigh $>8$ ) significantly enhances the contribution of passive galaxies in faint samples.

SF galaxies depends on environment. If the dominance of passive galaxies at high luminosity and the dominance of activeSF galaxies at low luminosity were independent of neighbour density, we would expect all panels in Fig. 3 to be similar. This is not the case, however, differences among panels are modest: whatever the number of satellites, bright samples are dominated by passive galaxies, and faint samples by active-SF ones.

Therefore, in general, luminosity dominates over neighbour multiplicity in setting the spectral-type mix of a galaxy population. A luminous galaxy might have few or many satellites, but will likely trace a deep potential. A faint galaxy might have few or many neighbours but will likely trace a shallow potential. It is only in extreme environments that the mix set by luminosity is significantly modified: galaxies with neigh $>8$ have their star-formation level (a typical active-SF one) suppressed even in faint samples, whereas isolated $($ neigh $=0)$ galaxies are still $40 \%$ likely to be star-forming (quiet-SF) at $M_{B}-$ $5 \log h_{75} \simeq-21.5$. These trends are consistent with result discussed in Sect. 3, namely that "minority" population can be identified in very luminous and very faint samples whose luminosities are inaccurate tracers of their halo mass: luminous SF galaxies trace small (sub-group size) halos, whereas faint passive galaxies trace massive (group/cluster size) halos.

Figures 2 and 3 also provide evidence that fractions of active-SF and quiet-SF galaxies exhibit distinct trends with luminosity: the fraction of active-SF galaxies decreases towards increasing luminosity, while the fraction of quietSF galaxies is nearly independent of luminosity, except for the most luminous samples. While Fig. 1 indicates that the dependence on density is the same for quiet-SF

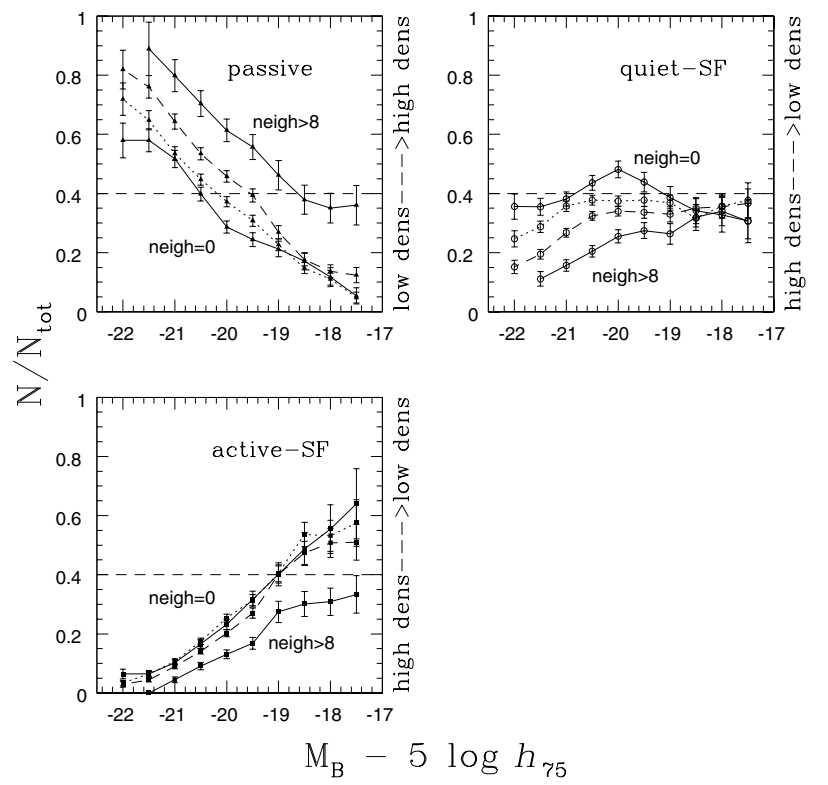

Fig. 4. Relative content in passive (top-left), quiet-SF (top-right) and active-SF (bottom-left) galaxies in samples of galaxies in different environments. Continuum lines are used to connect galaxy fractions with 0 and $>8$ neighbours, dotted and hatched lines to connect galaxy fractions in intermediate dense environments $(1 \leq$ neigh $\leq 2$ and $3 \leq$ neigh $\leq 8$ ).

and active-SF galaxies (Madgwick et al. 2003), Figs. 2 and 3 indicate that the dependence on luminosity is different. The data thus suggest a bimodal behaviour for galaxies with satellite-density and a "trimodal" behaviour (passive, quiet-SF, and active-SF) with luminosity. Bimodality in the distribution of galaxies properties has been addressed in many recent papers (Strateva et al. 2001; Hogg et al. 2002; Balogh et al. 2004b; Berlind et al. 2005; Blanton et al. 2005a).

\section{The role of satelite density for passive and SF galaxies}

To further explore the trend of increasing passive and decreasing SF galaxy fraction with luminosity and fainter neighbour density we also show, in Fig. 4, the fractional content of passive, quiet-SF and active-SF galaxies in different environments. The relative role of extremely dense and intermediate dense environment can be easily explored by comparing the gap between the neigh $=0$ and the neigh $>8$ lines with the the gap between the neigh $=0$ and the neigh $=3-8$ lines. Figure 4 shows that, for all 3 types, the gaps undergo a strong variation at magnitude $M_{B}-5 \log h_{75} \approx-19$. Therefore, we will keep the analysis of faint and bright samples separate.

Figure 4 shows that in luminous samples the fraction of isolated passive galaxies (neigh $=0$ ) is always below the fraction of passive galaxies with neigh $>8$, and that the gap does not depend on luminosity. A similar specular large gap is observed for quiet-SF galaxies, whereas the gap is smaller for active-SF galaxies. A smaller, but still significant, gap is observed between the neigh $=0$ and the neigh $=3-8$ lines, in passive and quiet-SF galaxy samples. The size of the gap is, again, nearly 
luminosity independent. Conversely, no gap is associated with active-SF galaxies.

In faint samples $\left(M-5 \log h_{75} \geq-19\right)$, passive and activeSF galaxies exhibit a large gap between the neigh $=0$ and the neigh $>8$ lines, whereas no gap is associated with the neigh $=0$ and neigh $=3-8$ transition. Again, a distinct behaviour is observed for active-SF and quiet-SF galaxies, the latter being equally frequent in all environments.

In summary, Fig. 4 indicates that a continuous parameterization of neighbour multiplicity (from 0 to $1-2$ to $3-8$ to $>8$ ) is indeed meaningful for bright samples, where neighbours are mainly satellites, as it relates to different fractions of passive and quiet-SF galaxies. In faint samples, however, a thresholdlike density parameterization appears to describe the galaxy behaviour better than a continuous one. This suggests that a continuous relation linking spectral-type with density only occurs when computing the density of satellites surrounding very luminous galaxies and implies that the spectral-type/density relation traces an enhanced correlation inside massive halos rather than an enhanced correlation between distinct halos.

The $M_{B}-5 \log h_{75} \simeq-19$ magnitude is a critical one: it corresponds to the luminosity at which the dependence on satellite-density moves from continuous to threshold-like, and also to the luminosity where fractions of active-SF galaxies become larger than the fractions of quiet-SF galaxies (see Fig. 2). Therefore it corresponds to the luminosity above which samples of passive galaxies exhibit a dependence on fainter neighbour density that is specular relative to that of quietSF galaxies, and below which passive galaxies are specular to active-SF galaxies. This is consistent with the finding (Norberg et al. 2002a; Balogh et al. 2004a) that, for low luminosity galaxies, clustering is a strong function of color, while for luminous galaxies clustering is a strong function of luminosity.

\section{The dependence on luminosity of the spectral-type/neighbour density relation}

We have shown that the spectral-type/density relation is possibly a spectral-type/satellite-density relation that traces an enhanced correlation inside single massive halos rather than enhanced correlation between distinct halos. To test this assumption directly we next examine the dependence of the spectraltype/density ( $\sim$ morphology/density) relation on luminosity. In Fig. 5 we show the spectral-type/density relation, with fractions of passive, quiet-SF, active-SF and all-SF galaxies normalized to the total number of galaxies in a given density bin, for 9 volume-limited samples. The faintest sample is not shown because it is small (see Table 2) and more affected by statistical uncertainties.

Figures 5 provides evidence that the fractional increase of passive galaxies between extreme densities is a universal characteristic of galaxies that does not depend on luminosity. What depends on luminosity is the fractional increase (decrease) of passive (SF) galaxies between neigh $=0$ and a group-like density (neigh $=3-8$ ). The increase (decrease) is not observed in samples fainter than $\simeq-19$. This supports our claim that the spectral-type/density relation is actually a process linked to the accretion of satellites by large massive halos, and not to enhanced correlation between distinct halos.

Figures 4 and 5 also indicate that the efficient formation of faint passive galaxies is a threshold process that only occurs in systems where a galaxy has a very large number of neighbours; it does not proceed gradually with neighbour density. Clearly this suggest that mechanisms acting only in clusters (ram pressure and stripping) are more efficient than those acting also in groups (galaxy interactions) in generating faint passive galaxies.

Finally Fig. 5 indicates that the neighbour density range corresponding to the intersection between early-type and latetype galaxies moves towards richer regions as the luminosity of the samples decreases. At $M_{B}-5 \log h_{75}=-22$ passive galaxies appear more numerous than SF galaxies even at the lowest densities $($ neigh $=0)$. At $M_{B}-5 \log h_{75}=-20$ equipartition is reached at group-like densities (neigh $=3-8$ ). At $M_{B}-5 \log h_{75}=-18$ equipartition is never reached; passive galaxies are no more than one-third of the population even in the densest environment (neigh $>8$ ). This confirms that, on the group scale, luminosity generally dominates over environment in setting the spectral type mix of a population. The result is complementary to the finding (Norberg et al. 2002a) that luminosity, and not type, is the dominant factor in determining how the clustering strength of the whole galaxy population varies with luminosity. However, because in luminous (faint) samples galaxies are mainly passive (active-SF), the correlation with type is strong at both the high and low luminosity end.

Given the correlation between spectral-type and morphology (Madgwick et al. 2002), Fig. 5 also likely illustrates a strong dependence of the morphology/density relation (Davis \& Geller 1976; Dressler 1980; Postman \& Geller 1984; Maia \& da Costa 1990; Helsdon \& Ponman 2003) on luminosity. Nevertheless, to prove this dependence for the morphology/density relation might be difficult as the correlation between environment and stellar age (color and spectral-type) appears stronger than the correlation between environment and morphology (Willmer et al. 1998; Kauffmann et al. 2004; Blanton et al. 2005a).

\section{Summary and conclusions}

In this paper we have investigated relations linking the spectraltype properties of $2 \mathrm{dF}$ galaxies to their luminosity and local neighbour density characteristics. We have assigned a local density to galaxies computing the number of neighbours within a $1 h_{75}^{-1} \mathrm{Mpc}$ projected distance and $\pm 1000 \mathrm{~km} \mathrm{~s}^{-1}$ depth. Our approach differs from previous analyses dealing with the same issue, because we have computed densities counting only fainter neighbours and applying a maximum magnitude difference criterion ( $\left.-2 \leq M_{\mathrm{gal}}-M_{\text {neigh }} \leq 0\right)$. This implies that, at least for luminous galaxies, neighbours likely trace the density of satellites that have been captured by the galaxy halo.

We have shown that the local density distribution for the alltype galaxy sample is approximately luminosity-independent over the whole explored luminosity range. This indicates that, at least on a $1 h_{75}^{-1} \mathrm{Mpc}$ scale, the number of neighbours associated with a galaxy is very similar, whether it reflects the 

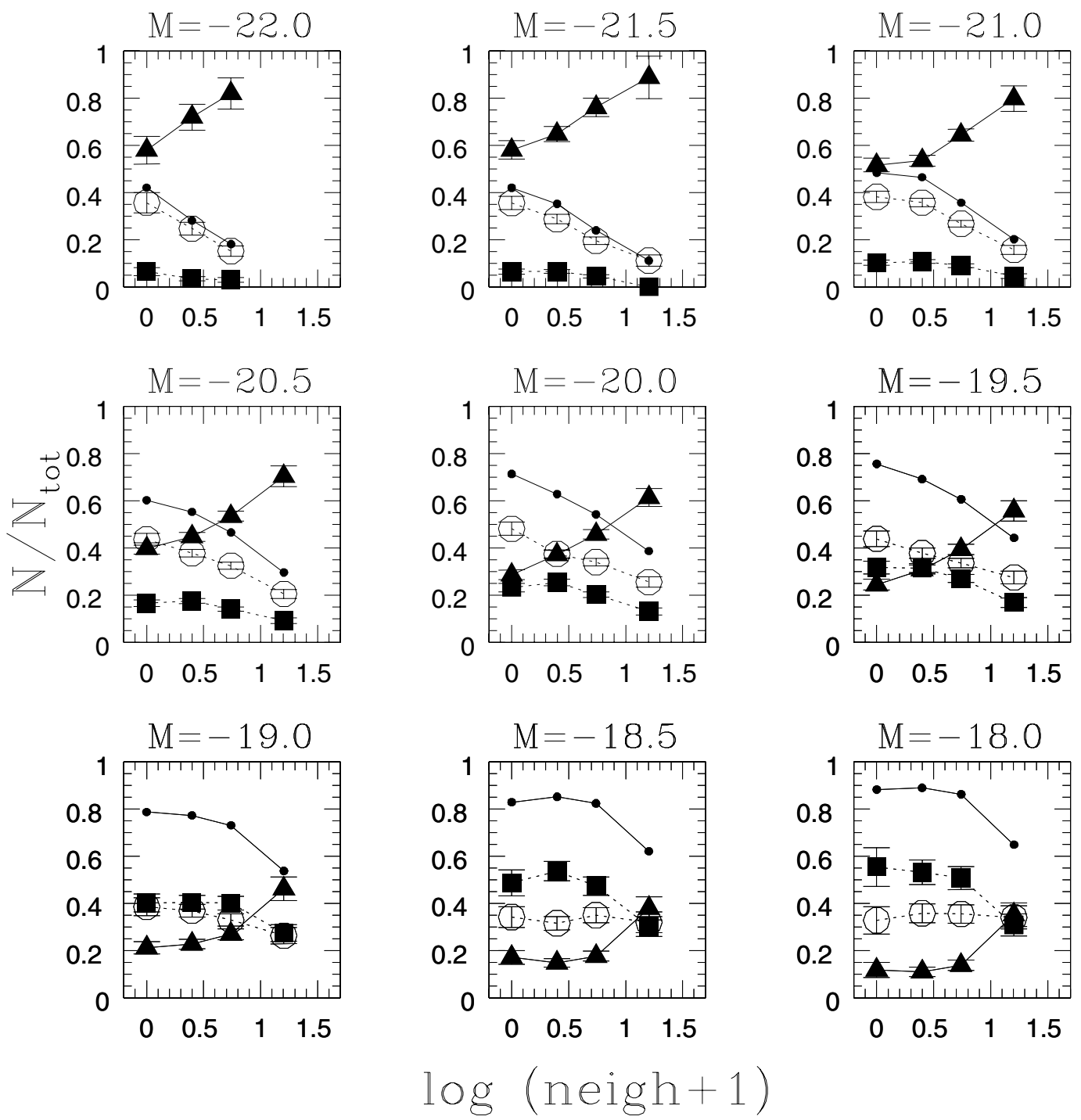

Fig. 5. The spectral-type/satellite-density relation for passive, quiet-SF and active-SF galaxies in the 9 brightest samples. Symbols are as in Fig. 2, small dots indicate the cumulative contribution of active and quiet-SF galaxies. Fractions have been computed using values listed in Table 2, normalized to the total number of galaxies in a given density bin so that, in each environment, the sum over all spectral types is 1.

number of satellites accreted by a luminous galaxy halo or the number of neighbours of low luminosity galaxies still in their original small halos.

We have also found that that the excess of fainter companions surrounding passive galaxies is not limited to luminous galaxies (Norberg et al. 2002a; Hogg et al. 2003; Berlind et al. 2005; Blanton et al. 2005a) but is instead a general characteristic of passive galaxies. The stronger clustering of passive galaxies relative to SF galaxies, on the group scale, appears to arise from two distinct contributions. At the luminous end, it is due to an excess of satellites surrounding central galaxies inside large halos. At the faint end it is due to an excess of satellites that are strongly correlated among themselves.

We have shown that the global spectral-type/luminosity relation (Fig. 2) is not significantly altered in subsamples exhibiting different satellite densities. Whatever the environment, passive galaxies (type 1) numerically dominate in luminous samples, and active-SF galaxies (type $3+4$ ) numerically dominate in faint ones. In contrast, the relative content in quiet-SF galaxies shows a weak dependence on luminosity. Only galaxies in extreme environments exhibit significant departures from these general trends: in dense environments (neigh $>8$ ) a significant fraction of passive galaxies is observed even among faint galaxies, whereas among isolated galaxies (neigh $=0$ ), quiet-SF (type 2) galaxies still represent a $40 \%$ fraction of the luminous population. We suggest that these "minority" populations, identified in very luminous and very faint samples, are poor tracers of halo mass: luminous SF galaxies are actually tracers of small (sub-group scale) halos, whereas faint passive galaxies are tracer of massive (group/cluster scale) halos.

Our analysis provides evidence for the existence of a global spectral-type/satellite-density relation, with the fraction of passive galaxies steadily growing (and the fraction of quiet-SF galaxies steadily decreasing) when moving from an isolated galaxy sample to galaxies with cluster-like neighbour density. But we have also shown that this relation only holds in luminous samples; in faint samples the variation in the fractional content of passive (SF) between the neigh $=0$ and the 
intermediate dense (neigh $=3-8$ ) environments is not observed: the dependence on environment becomes threshold-like, and very dense environments are required to observe a variation in the spectral-type mix. This suggests that the morphology/density relation is likely a morphology/satellite-density relation, that traces enhanced correlation inside single massive halos rather than enhanced correlation between distinct halos.

Acknowledgements. We thank A. Berlind, A. Biviano, R. De Propris, T. Goto and C.N.A. Willmer for comments and suggestions. We are also indebted to the anonymous referee whose comments and criticism greatly improved the scientific content of this paper. This work was supported by MIUR, B.K. acknowledges a fellowship from Bologna University.

\section{References}

Balogh, L. M., Eke, V., Miller, C. J., et al. 2004a, MNRAS, 348, 1355

Balogh, L. M., Baldry, I. K., Nichol, R., et al. 2004b, ApJ, 615, 101

Berlind, A. A., Blanton, M. R., Hogg, D. W., et al. 2005, ApJ, 629, 625

Blanton, M. R., Eisenstein, D. J., Hogg, D. W., et al. 2005a, ApJ, 629, 143

Blanton, M. R., Eisenstein, D. E., Hogg, D. W., \& Zehavi, I. 2005b, ApJ, submitted [arXiv: astro-ph/0411037]

Christlein, D., \& Zabludoff, A. I. 2005, ApJ, 621, 201

Colless, M. M., \& the 2dFGRS team 2001, MNRAS, 328, 1039

Colless, M. M., \& the 2dFGRS team 2003 [arXiv:astro-ph/0306581]

Davis, M., \& Geller, M. J. 1976, ApJ, 208, 13

Dominguez, M. J., Zandivarez, A. A., Martinez, H. J., et al. 2002, MNRAS, 335, 825

Dressler, A. 1980, ApJ, 236, 351

Eke, V. R., Baugh, C. M., Cole, S., \& the 2dFGRS team 2004a, MNRAS, 348, 866

Eke, V. R, Frenk, C. S., Baugh, C. M., \& the 2dFGRS team 2004b, MNRAS, 348, 1355

Folkes, S., Ronen, S., Price, J., et al. 1999, MNRAS, 308, 459

Gerken, B., Ziegler, B. L., Balogh, M. L., et al. 2004, A\&A, 421, 59

Gomez, P. L., Nichol, R. C., Miller, C. J., et al. 2003, ApJ, 584, 210

Goto, T., Hokamura, S., McKay, T., et al. 2002, PASJ, 54 , 515
Goto, T., Yamauchi, C., Fujita, Y., et al. 2003, MNRAS, 346, 601

Helsdon, S. F., \& Ponman, T. J. 2003, MNRAS, 339, L29

Hogg, D. W., Blanton, M., Strateva, I., et al. 2002, AJ, 124, 646

Hogg, D. W., Blanton, M. R., Eisenstein, D. J., et al. 2003, ApJ, 585, L5

Hubble, E., \& Humason, M. L. 1931, ApJ, 74, 43

Jing, Y. P., \& Borner, G. 2004, ApJ, 617, 782

Kauffmann, G., White, S. D. M., Heckman, T. M., et al. 2004, MNRAS, 353, 713

Kelm, B., \& Focardi, P. 2004a, A\&A, 418, 937

Kelm, B., \& Focardi, P. 2004b, in Outskirts of galaxy clusters: intense life in the suburbs, ed. A. Diaferio, IAU Coll., 195, 456

Lewis, I., Balogh, M., De Propris, R., et al. 2002, MNRAS, 334, 673

Maddox, S. J., Efstathiou, G., \& Sutherland, W. J. 1990, MNRAS, 246, 433

Madgwick, D. S., Lahav, O., Baldry, I. K., et al. 2002, MNRAS, 333, 133

Madgwick, D. S., Hawkins, E., Lahav, O., et al. 2003, MNRAS, 344, 847

Maia, M. A. G., \& da Costa, L. N. 1990, ApJ, 352, 457

Mateus, A. J., \& Sodré, L. J. 2004, MNRAS, 349, 1251

Merchán, M., \& Zandivarez, A. 2002, MNRAS, 335, 216

Norberg, P., Baugh, C. M., Hawkins, E., et al. 2002a, MNRAS, 332, 827

Norberg, P., Baugh, C. M., Hawkins, E., et al. 2002b, MNRAS, 336, 907

Mulchaey, J. S., Davis, D. S., Mushotzky, R. F., \& Burstein, D. 2003, ApJS, 145, 39

Postman, M., \& Geller, M. J. 1984, ApJ, 281, 95

Strateva, I., Zeljko, I., Knapp, G. R., et al. 2001, AJ, 122, 1861

Tanaka, M., Goto, T., Sadanori, O., Shimasaku, K., \& Brinkmann, J. 2004, ApJ, 128, 2677

Tran, K. H., Simard, L., Zabludoff, A. I., \& Mulchaey, J. S. 2001, ApJ, 549,172

Whitmore, B. C. 1995, in Groups of Galaxies, ed. O. G. Richter, \& K. Borne, ASP Conf. Ser., 70, 41

Whitmore, B. C., \& Gilmore, D. M. 1991, ApJ, 367, 64

Whitmore, B. C., Gilmore, D. M., \& Jones, C. 1993, ApJ, 407, 489

Willmer, C. N. A., da Costa, L. N., \& Pellegrini, P. S. 1998, AJ, 115, 869

Zehavi, I., Blanton, M. R., Frieman, J. A., et al. 2002, ApJ, 571, 172

Zehavi, I., Zheng, Z., Weinberg, D. H., et al. 2005, ApJ, 630, 1 